\title{
Malformación genital: disparador del diagnóstico de variantes severas de síndrome de Klinefelter
}

\section{Genital malformation: trigger of the diagnosis of severe variants of Klinefelter syndrome}

\author{
Fadil Iturralde, José Luis ${ }^{\mathrm{a}}$; Marani, Jorge ${ }^{\mathrm{a}}$; Lahoz García, Martha ${ }^{\mathrm{b}}$; Carbognani, Silviac; \\ Luccerini, Verónicac; Damiani, Horacio Juan ${ }^{\mathrm{a}}$; Contardi, Juan Carlos ${ }^{\mathrm{a}}$
}

\author{
aServicio de Urología Pediátrica, Sanatorio de Niños de Rosario. Grupo Oroño. Argentina \\ 'servicio de Clínica Pediátrica, Sanatorio de Niños de Rosario. Grupo Oroño. Argentina \\ cServicio de Genética Clínica, Sanatorio de Niños de Rosario. Grupo Oroño. Argentina
}

Recibido: 29 de mayo de 2019; Aceptado: 8 de septiembre de 2019

\section{¿Qué se sabe del tema que trata este estudio?}

El Síndrome de Klinefelter clásico es ampliamente conocido. Sus variantes son infrecuentes y se asocian a trastornos profundos del desarrollo neurocognitivo además de las malformaciones genitales. Estos pacientes raramente son diagnosticados antes de la segunda infancia.

\section{¿Qué aporta este estudio a lo ya conocido?}

La sospecha de alteración genética en niños con malformación genital favoreció el diagnóstico precoz de variantes severas de Síndrome de Klinefelter, permitiendo una rápida intervención del equipo de salud para mitigar los trastornos neurocognitivos y de sociabilización presentes en estos niños.

\section{Resumen}

El síndrome de Klinefelter y sus variantes, como alteración en el número de cromosomas sexuales, se encuentra entre los trastornos del desarrollo sexual. Sus portadores manifiestan hipogonadismo hipergonadotrófico en la pubertad; las variantes severas presentan además problemas neurocognitivos y del lenguaje desde edades tempranas. Objetivo: Describir dos pacientes portadores de malformación genital con diagnóstico genético de variantes severas de síndrome de Klinefelter; y revisar aspectos clínicos y terapéuticos. Casos Clínicos: Caso 1: Diagnóstico de genitales atípicos al nacer: Falo pequeño y corvo con meato uretral a nivel escrotal y escroto bífido. Sin otra anomalía somática, excepto sutil clinodactilia del 5 dedo. Cariotipo: 49,XXXXY. Al año de vida se reconstruyeron los genitales. Evolucionó con retraso global del desarrollo, principalmente del lenguaje, manejado con estimulación temprana kinésica y fonoaudiológica desde los 2 meses, logró integrarse en un jardín de infantes. Caso 2: $\mathrm{Al}$ mes de vida se constató falo pequeño y corvo severo (más de $70^{\circ}$ ), testículos en bolsa. Cariotipo: 48,XXYY. Al año de vida se corrigió malformación del pene. Evolucionó con retraso global del desarrollo, fundamentalmente en el lenguaje expresivo, y fue manejado con el equipo de estimulación temprana desde los 4 meses, logrando adaptación en un jardín de infantes. Conclusión: Las malformaciones genitales condujeron al diagnóstico de variantes severas de síndrome de Klinefelter, y fueron corregidas alrededor del año de vida. La identificación temprana de estas variantes permitió la intervención del equipo de neuroestimulación, favoreciendo el desarrollo neurocognitivo y la integración social de estos niños.

Palabras clave: Síndrome de Klinefelter; malformación genital; 48,XXYY; 49,XXXXY; hipogonadismo 


\begin{abstract}
Among the disorders of sexual development, Klinefelter syndrome and its variants are classified as an alteration in the number of sex chromosomes. These patients show signs of hypergonadotropic hypogonadism at puberty, however cases of severe variants also present neurocognitive and language problems from an early age. Objective: To describe two patients with genital malformation with genetic diagnosis of severe variants of Klinefelter syndrome, and to review clinical and therapeutic aspects. Clinical Cases: Case 1: Diagnosis of atypical genitalia at birth: Small and curved phallus with the urethral meatus at scrotal level, and bifid scrotum. No other somatic abnormality was observed, except for subtle clinodactyly of the fifth finger. Karyotype: 49 , XXXXY. At one year of life, genitalia were reconstructed. The patient presented a global developmental delay, mainly in language, which was managed with early stimulation and speech and language therapy since he was two months old. Finally, he was able to attend kindergarten. Case 2: At one month of life, a small and severe curved phallus (more than $70^{\circ}$ ) was observed, and testicles were in the scrotum. Karyotype: 48, XXYY. At one year of life, the penile malformation was corrected. The patient presented global developmental delay, mainly in expressive language which was managed with early stimulation since the age of four months, achieving kindergarten attendance. Conclusion: Genital malformations led to the diagnosis of severe variants of Klinefelter syndrome, and were corrected around the year of life. The early identification of these variants allowed the intervention of the neurostimulation team, favoring the neurocognitive development and social integration of these children.
\end{abstract}

\section{Keywords:}

Klinefelter syndrome; genital malformation; 48,XXYY; 49,XXXXY; hypogonadism

\section{Introducción}

Ante la consulta de un paciente con alteraciones genitales severas, es imprescindible la realización de una evaluación genética, dado que en ocasiones pueden detectarse síndromes que presentan patologías asociadas de gravedad variable ${ }^{1,2}$. De confirmarse esta circunstancia, el paciente deja de ser sólo portador de una malformación genital y se convierte en un paciente sindrómico, con múltiples connotaciones para su salud, con la necesidad de un manejo multidisciplinario especializado. La omisión o el retardo en el diagnóstico podría tener implicancias pronósticas relevantes en el futuro de ese niño ${ }^{2,3}$.

En 2005 se celebró una conferencia de consenso que condujo al desarrollo de una nueva terminología que ha sido ampliamente adoptada. Utiliza el término "trastornos del desarrollo sexual" (DSD abreviatura en inglés) para describir la amplia gama de afecciones involucradas en el desarrollo genital y sexual; y las clasifica en base a la constitución cromosómica de los pacientes ${ }^{4,5}$.

El síndrome de Klinefelter y sus variantes constituyen un trastorno del desarrollo sexual resultante de una alteración en el número de cromosomas sexuales. Se la considera como la anomalía de mayor frecuencia en los seres humanos, y se caracteriza por la presencia de al menos un cromosoma $\mathrm{X}$ adicional al cariotipo masculino normal XY. La variante clásica $(47, X X Y)$ es la más frecuente, con una incidencia de 1 en 650 varo$n^{2}{ }^{2}$. En cambio, las variantes severas son extremadamente inusuales: los pacientes 48,XXXY tienen una in- cidencia de 1:18.000-1:40.000; los pacientes 48,XXYY una incidencia de 1:50.000; y los pacientes 49,XXXXY una incidencia de 1:100.000) ${ }^{6}$.

Los niños portadores de estas variantes raras del síndrome de Klinefelter, además de presentar signos de hipogonadismo hipergonadotrófico en la pubertad, presentan problemas neurocognitivos y en el lenguaje que se manifiestan a partir de los 18 meses de vida ${ }^{3,6}$. El diagnóstico precoz en estos niños es fundamental para iniciar terapia de neuroestimulación temprana y mejorar su pronóstico a largo plazo.

Es excepcional la presencia de malformaciones genitales severas que lleven al diagnóstico temprano de síndrome de Klinefelter, circunstancia que motivo esta revisión ${ }^{6,7}$.

El objetivo de esta comunicación es describir dos pacientes portadores de malformación genital con diagnóstico genético de variantes severas de síndrome de Klinefelter, y revisar aspectos clínicos y terapéuticos.

\section{Casos Clínicos}

\section{Caso 1}

Segundo hijo de padres no consanguíneos, sin antecedentes mórbidos importantes, con diagnóstico prenatal ecográfico de embarazo único, de sexo femenino y sin patología aparente. Parto eutócico de 39 semanas de edad gestacional. $\mathrm{Al}$ nacer se pesquisaron genitales atípicos, por lo que se solicitó interconsulta con el Servicio de Urología.

$\mathrm{Al}$ examen físico se constató la presencia de un 
falo pequeño, corvo, con meato uretral a nivel escrotal, trasposición penoescrotal y escroto bífido. Ambos testículos de tamaño habitual para la edad en bolsas (figura 1). Grado virilización externa: 3 (clasificación adaptada por Ahmed y cols. $)^{8}$. Clinodactilia muy sutil del $5^{\circ}$ dedo mano derecha; sin otras alteraciones al examen físico. La ecografía abdominal no evidenció signos de estructuras Müllerianas. Evaluado por el Servicio de Genética, se informó cariotipo sexo masculino con polisomia sexual 49,XXXXY. Se descartaron trastornos neurológicos y cardiovasculares significativos. A los 10 meses empezó a observarse un cambio en su facie: fisuras palpebrales elevadas, hipertelorismo ocular, y puente nasal plano. Al partir del año de vida se reconstruyeron los genitales en 2 tiempos quirúrgicos, lográndose resultados funcionales y estéticos satisfactorios.

En cuanto a su desarrollo, a los 2 meses de vida inició tratamiento con estimulación temprana kinésica y fonoaudiológica. En la evaluación a los 3 años de vida se describió un retraso global del desarrollo determinado a través de la evaluación realizada con el Instrumento para la Observación del Desarrollo Infantil. Ministerio de Salud de la República Argentina (IODI) ${ }^{9}$, principalmente en el área del lenguaje, con buena comprensión y muy poca fluencia verbal. Sin embargo, el apoyo del equipo de estimulación temprana le permitió la integración en un jardín de infantes, dando lugar a la inclusión social del paciente.

\section{Caso 2}

Padres sanos, no consanguíneos, que realizaron tratamiento de fertilización asistida (ICSI) mediante ovodonación por infertilidad. Las ecografías prenatales informaban feto único de sexo masculino sin alteraciones. Parto eutócico en semana 38, con evolución perinatal sin particularidades. Al mes de vida la pediatra notó "pene atípico" y efectuó interconsulta con urólogo pediátrico, quien constató falo pequeño, corvo severo (más de $70^{\circ}$ ) con meato aparente a nivel del glande y muy escasa piel ventral. Escroto de características normales con ambos testículos en bolsa, siendo el derecho de menor tamaño (figura 2). Grado virilización externa: $7^{8}$. No se observaron otras dismorfias de significación. El Servicio de Genética informó niño de sexo masculino con polisomia sexual con cariotipo: 48,XXYY. Ecografía abdominal sin particularidades y sin evidencia de estructuras Müllerianas. Al año de vida se corrigieron genitales quirúrgicamente, logrando un pene recto con meato de aspecto y ubicación normal en glande.

En cuanto al desarrollo, a los 4 meses de vida comenzó con estimulación temprana kinésica y fonoaudiológica. En la evaluación a los 2 años de edad presentaba un retraso global del desarrollo determinado por evaluación IODI, con buena comprensión y dificultad en el lenguaje expresivo verbal. Inició su integración en un jardín de infantes asistido por acompañante terapéutico.

\section{Discusión}

Los síndromes 48,XXYY; 48,XXXY y 49,XXXXY son anomalías de aneuploidía cromosómica sexual que conducen a disgenesia testicular, y se manifiestan

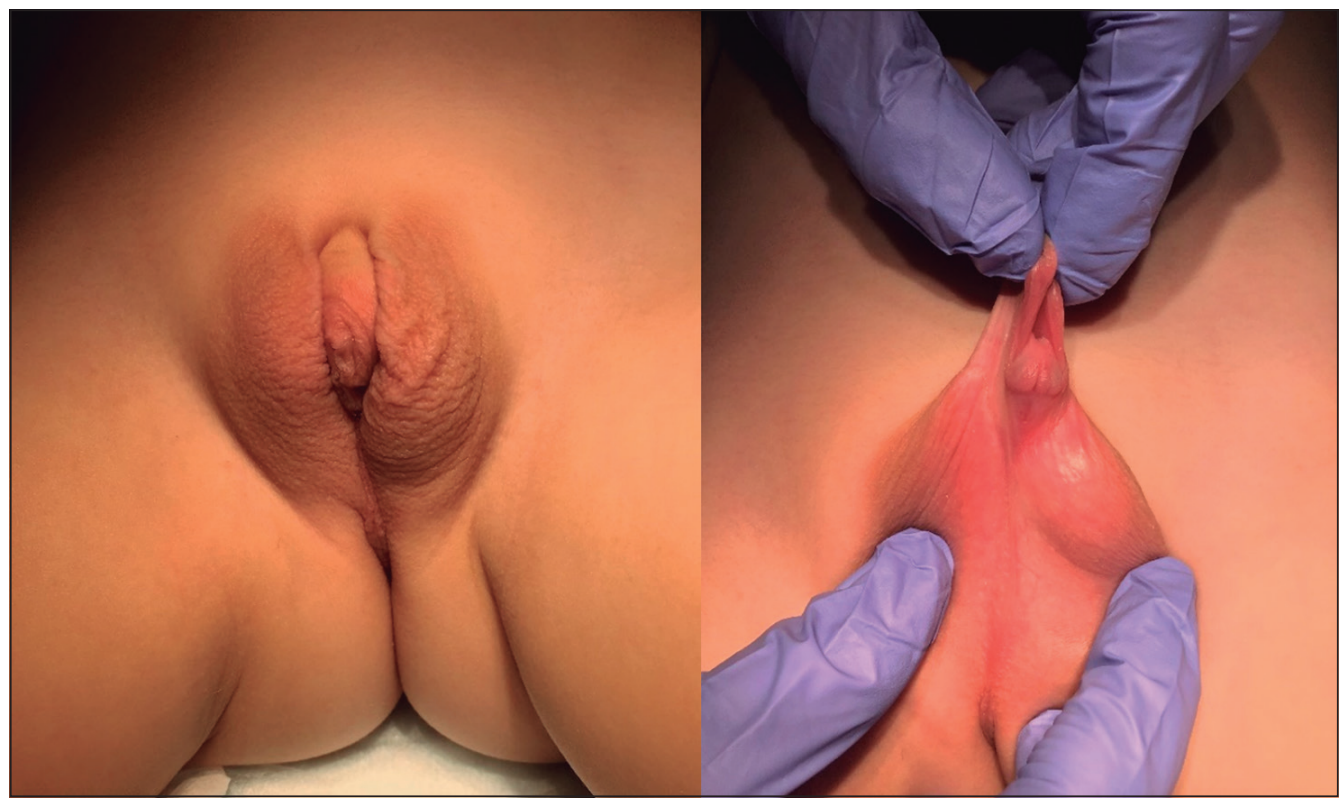

Figura 1. Aspecto de genitales externos del paciente con síndrome 49, XXXXY. 


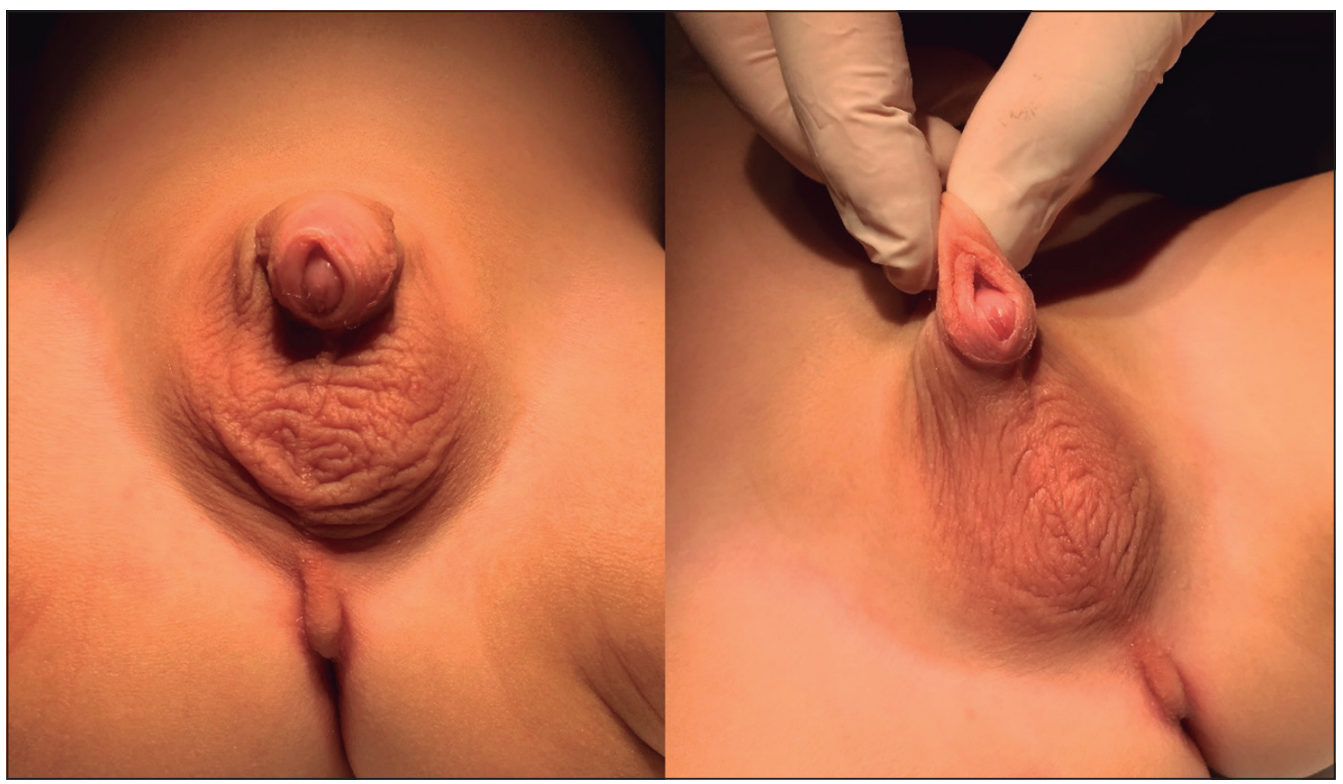

Figura 2. Aspecto de genitales externos del paciente con síndrome 48, XXYY.

a través de alteraciones en la esfera genital y reproductiva (dependiendo del grado de hipogonadismo). Esta observación ha hecho que se los considere como variantes del síndrome de Klinefelter. No obstante, estos pacientes manifiestan además retardo en el desarrollo neurológico y cognitivo, trastornos en el habla, problemas de comportamiento y socialización, y diversas malformaciones esqueléticas; suscitando dudas sobre la consideración de estos trastornos como variantes del síndrome de Klinefelter clásico ${ }^{6,10,11}$.

En general, los pacientes 48,XXYY al nacer no muestran rasgos distintivos característicos. Aisladamente, como en el caso 2, presentan alteraciones genitales que pueden variar desde una criptorquidia o hidrocele comunicante, hasta severas malformaciones con penes pequeños, hipospádicos o corvos. Otros signos físicos pueden ser: clinodactilia del $5^{\circ}$ dedo (en el $70 \%$ de los casos), pie plano, problemas dentarios, hipertelorismo con fisuras palpebrales hacia arriba y epicantus. Rara vez presentan cardiopatías ${ }^{6,12}$. Se describe también como parte del síndrome la sinostosis radiocubital y la alergia alimentaria o respiratoria ${ }^{11}$.

Si no presentan alteraciones genitales ni esqueléticas, los primeros síntomas aparecen luego de los 18 meses de edad, con hipotonía leve en el 75\% de los casos. También pueden mostrar retardo en el desarrollo del lenguaje (sobre todo expresivo) y distintos grados de retraso psicomotriz y cognitivo. Todos tienen dificultad en el aprendizaje en la edad escolar, con un coeficiente intelectual de alrededor de 70-80. En cuanto al desarrollo social, en general son dóciles y sumisos, pero se han descriptos casos de agresividad extrema $a^{6,13}$.

El síndrome 49,XXXXY fue reportado por primera vez en 1960 por Fraccaro et al. ${ }^{14}$ Se lo conoce como la variante más severa del síndrome de Klinefelter debido a la presencia de hipogonadismo hipergonadotrófico asociado a una combinación de alteraciones esqueléticas, neurológicas, cognitivas, malformaciones genitales y cardiacas.

Son muy limitados los datos publicados sobre pacientes recién nacidos con síndrome 49,XXXXY. La presencia de varias anomalías congénitas no especificas han llevado al diagnóstico de este síndrome en edades tempranas: bajo peso al nacer, microcefalia, úvula bífida, paladar hendido, hipotonía muscular, clinodactilia del quinto dedo, ductus arteriovenoso persistente, o malformaciones en genitales ${ }^{15}$. Los rasgos faciales son un signo casi patognomónico (hipertelorismo ocular, levantamiento de fisuras palpebrales y puente nasal plano) pero no es posible percibirla hasta los 9-12 meses de edad ${ }^{3,16}$. Los fenotipos cambian a medida que el niño crece hacia la adultez, por lo tanto, ciertas características clínicas de los postpúberes no son claras en los niños menores ${ }^{7}$. Son pacientes de baja talla (a diferencia del resto de las variantes) y pueden presentar otras alteraciones esqueléticas: sinostosis radiocubital, genu valgum, pie cavo, escoliosis, hipotonía con articulaciones laxas.

El coeficiente intelectual oscila habitualmente entre 20 y 60, con gran dificultad para el aprendizaje. El retraso cognitivo y del desarrollo del lenguaje afecta seriamente la socialización de estos niños. Tienden a ser tímidos, amistosos, con irritabilidad ocasional y rabietas, baja tolerancia a la frustración y dificultad para adaptarse al cambio de rutinas.

Se cree que la variabilidad en las características clí- 
nicas de estos síndromes deriva de la manifestación de tres factores: la gravedad del defecto genético (números de X), la deficiencia de andrógenos, y la sensibilidad del receptor de andrógenos. Cuanto más alterados se encuentran estos factores, mayor será la severidad de la expresión fenotípica ${ }^{1,2}$.

\section{Conclusión}

En los casos clínicos presentados, la presencia de una malformación genital severa hizo sospechar la existencia de algún trastorno genético subyacente, que fue confirmada con la evaluación clínica y genómica.

Las malformaciones genitales fueron el signo disparador del diagnóstico de variantes severas de síndrome de Klinefelter, y se resolvieron en torno al año de vida por el Servicio de Urología. La identificación temprana de estos síndromes posibilitó la pronta intervención del equipo de estimulación temprana (fonoaudiólogos, kinesiólogos, psicopedagogos, neurólogos, fisiatras, etc.), promoviendo el desarrollo neurocognitivo y fonoaudiológico de estos pacientes, con un impacto positivo en su integración social.

\section{Responsabilidades Éticas}

Protección de personas y animales: Los autores declaran que los procedimientos seguidos se conformaron a las normas éticas del comité de experimentación humana responsable y de acuerdo con la Asociación Médica Mundial y la Declaración de Helsinki.

Confidencialidad de los datos: Los autores declaran que han seguido los protocolos de su centro de trabajo sobre la publicación de datos de pacientes.

Derecho a la privacidad y consentimiento informado: Los autores han obtenido el consentimiento informado de los padres (tutores) de la paciente y/o sujetos referidos en el artículo. Este documento obra en poder del autor de correspondencia

\section{Conflicto de intereses}

Los autores declaran no tener conflicto de intereses.

\section{Referencias}

1. Visootsak J, Graham JM Jr. Klinefelter syndrome and other sex chromosome aneuploidies. Orphanet J Rare Dis. 2006;1:42.

2. Bird RJ, Hurren BJ. Anatomical and clinical aspects of Klinefelter's syndrome. Clin Anat. 2016;29(5):606-19.

3. Gropman AL, Rogol A, Fennoy I, Sadeghin T, et al. Clinical variability and novel neurodevelopmental findings in 49,XXXXY syndrome. Am J Med Genet. 2010;152A:1523-30.

4. Lee PA, Houk CP, Ahmed SF, Hughes IA, et al. Consensus Statement on Management of Intersex Disorders. Pediatrics. 2006;118:488-500.

5. Hutson JM, Grover SR, O'connell M, Pennell SD. Malformation syndromes associated with disorders of sex development. Nat Rev Endocrinol 2014;10:476-87.

6. Tartaglia N, Ayari N, Howell S,
D'Epagnier C, et al. 48,XXYY, 48,XXXY and 49,XXXXY syndromes: not just variants of Klinefelter syndrome. Acta Paediatr 2011;100:851-60.

7. Etemadi K, Basir B, Ghahremani S. Neonatal diagnosis of 49, XXXXY syndrome. Iran J Reprod Med. 2015;13(3):181-4.

8. Ahmed SF, Khw Aja O, Hughes IA. The role of a clinical score in the assessment of ambiguous genitalia. BJU International 2000;85:120-4.

9. IODI. Instrumento para la observación del desarrollo infantil. Ministerio de Salud de la República Argentina. 2017 http:// www.msal.gob.ar/images/stories/bes/ graficos/0000000844cnt-iodi-variables-ybibliografia.pdf.

10. Peet J, Weaver D, Vance G. 49,XXXXY: a distinct phenotype. Three new cases and review. J Med Genet 1998;35:420-4.

11. Tartaglia N, Davis S, Hench A, Nimishakavi S, et al. A new look at XXYY syndrome: medical and psychological features. Am J Med Genet A 2008;146A:1509-22.

12. Zelante L, Piemontese MR, Francioli G, Calvano S. Two 48,XXYY patients: clinical, cytogenetic and molecular aspects. Ann Genet. 2003;46:479-81.

13. Atik T, Çoğulu O, Özkınay F. A rare sex chromosome aneuploidy: 48,XXYY sindrome. Turk Pediatri Ars 2016;51:1069.

14. Fraccaro M, Kaijser K, Lindsten J. A child with 49 Chromosomes. Lancet. 1960;2:899-902.

15. Kidszun A, Fuchs AJ, Russo A, Bartsch M, et al. Skeletal abnormalities of the upper limbs- Neonatal diagnosis of 49,XXXXY syndrome. Gene. 2012;508:117-20.

16. Sprouse C, Tosi L, Stapleton E, Gropman AL, et al. Musculoskeletal anomalies in a large cohort of boys with 49,XXXXY. Am J Med Genet C Semin Med Genet. 2013;163:44-9. 